Disponível em

http://www.anpad.org.br/rac

RAC, Curitiba, v. 15, n. 5, art. 2,

pp. 815-833, Set./Out. 2011

$(c)$ EY-NG

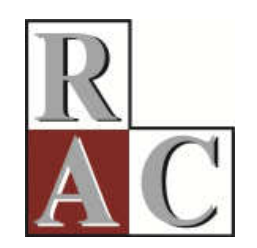

\title{
Resiliência e Socialização Organizacional entre Servidores Públicos Brasileiros e Noruegueses
}

\section{Resilience and Organizational Socialization among Brazilian and Norwegian Civil Servants}

\author{
Virgínia Donizete de Carvalho * \\ E-mail: vircah@gmail.com \\ Universidade Federal de Alfenas - UNIFAL \\ Varginha, MG, Brasil. \\ Livia de Oliveira Borges \\ E-mail: liviadeoliveira@gmail.com \\ Universidade Federal de Minas Gerais - UFMG \\ Belo Horizonte, MG, Brasil. \\ Arne Vikan \\ E-mail: arne.vikan@svt.ntnu.no \\ Universidade Norueguesa de Ciência e Tecnologia \\ Trondheim, Noruega. \\ Odin Hjemdal \\ E-mail: odin.hjemdal@svt.ntnu.no \\ Universidade Norueguesa de Ciência e Tecnologia \\ Trondheim, Noruega.
}

* Endereço: Virgínia Donizete de Carvalho

UNIFAL, Rua Alfredo Braga de Carvalho, 303, Parque Industrial JK, Varginha/MG, 37062-440.

Copyright (C) 2011 RAC. Todos os direitos, até mesmo de tradução, são reservados. É permitido citar parte de artigos sem autorização prévia, desde que seja identificada a fonte. 


\title{
Resumo
}

O estudo objetivou analisar a relação entre a resiliência e a socialização organizacional junto a novos servidores de duas universidades públicas, em contextos culturais distintos, quais sejam Brasil e Noruega. As pesquisas em socialização organizacional apontam que a adaptação do indivíduo a um novo cargo e a uma nova organização tende a ser um evento estressor e a resiliência, que descreve os processos de superação do estresse e adversidades, poderia auxiliar a compreensão das diferenças nos resultados de socialização entre indivíduos numa mesma organização. Com base nesse presssuposto, a pesquisa foi desenvolvida junto a uma amostra de servidores docentes e técnico-adiministrativos brasileiros e noruegueses. Análises de regressão hierárquica foram desenvolvidas com o intuito de observar e comparar a capacidade preditiva da resiliência em relação à socialização organizacional. Os resultados demonstraram, de modo geral, que a resiliência contribuiu significativamente para explicar os resultados de socialização organizacional, independentemente da nacionalidade e da ocupação. A capacidade preditiva da resiliência em relação à socialização organizacional foi maior entre os novos servidores brasileiros. As implicações práticas, limitações e principais contribuições do estudo são discutidas, com sugestões para futuras pesquisas.

Palavras-chave: resiliência; socialização organizacional; servidores públicos.

\begin{abstract}
The aim of this study is to analyze the relationship between resilience and organizational socialization among civil servants at two public universities in two different cultures, namely Brazil and Norway. Organizational socialization research has shown that an individual's adjustment to a new job and organization tends to be a stressful event and resilience, defined as the ability to overcome stress or adversity, could be of importance when it comes to explaining the differences in socialization outcomes among individuals at the same organization. Based on this assumption, research was conducted with a sample of Brazilian and Norwegian professors and technical-administrative employees. Hierarchical regression analyses were carried out to observe and compare the predictive power of resilience in relation to organizational socialization. The results showed that overall resilience added a significant incremental prediction to organizational socialization, beyond nationality and occupation. The predictive power of resilience in relation to organizational socialization was higher among the Brazilians than the Norwegians. The practical implications, limitations and main contributions of the study are discussed, with suggestions for future research.
\end{abstract}

Key words: resilience; organizational socialization; civil servants. 


\section{Introdução}

A socialização organizacional, que foca o processo de adaptação a um novo cargo e a uma nova realidade organizacional, tem-se mostrado relevante, à medida que se consideram as suas implicações para o desempenho, retenção de empregados e atitudes no trabalho (Bauer, Morrison, \& Callister, 1998). Uma tendência das pesquisas nesse campo tem sido a investigação do papel das diferenças individuais como variáveis preditoras, moderadoras ou mediadoras nos processos de socialização (Cooper-Thomas \& Anderson, 2006; Saks \& Ashforth, 1997). A autoeficácia, por exemplo, tem sido uma das variáveis mais empregadas nestes estudos (Gruman, Saks, \& Zweig, 2006; Saks, 1995), mas também já foram estudadas as experiências prévias de trabalho (Beyer \& Hannah, 2002; Carr, Pearson, Vest, \& Boyar, 2006), as dimensões de personalidade relacionadas à extroversão e à abertura a experiências (Wanberg \& Kammeyer-Mueller, 2000) e as variáveis demográficas (Finkelstein, Kulas, \& Dages, 2003), dentre outras.

Até o momento não foram identificadas, contudo, pesquisas que tenham examinado a resiliência como um fenômeno que afeta o processo de socialização organizacional, embora estudo recente (Hjemdal, Friborg, Stiles, Rosenvinge, \& Martinussen, 2006) demonstre seu poder explicativo, no que concerne às respostas dadas pelos indivíduos na vivência de acontecimentos considerados estressantes. Sabendo-se que o processo de adaptação do indivíduo a uma nova realidade organizacional constitui um evento gerador de estresse, dado que tais transições são normalmente acompanhadas de elevadas expectativas e sentimentos de incerteza (Bauer, Bodner, Erdogan, Truxillo, \& Tucker, 2007), considera-se a relevância de analisar a ação dos mecanismos de resiliência em tais circunstâncias. Entende-se que estes podem auxiliar a compreensão das diferenças nos resultados de socialização organizacional entre indivíduos que vivenciaram esse processo numa mesma organização.

Diante do exposto, o objetivo deste estudo foi analisar a relação entre a resiliência e a socialização organizacional de novos servidores em duas universidades públicas, pertencentes a contextos culturais distintos, quais sejam o Brasil e a Noruega. Há algum tempo, estudiosos têm apontado a conveniência da adoção de desenhos transculturais nas pesquisas (Bauer \& Taylor, 2001; Miller-Loessi \& Parker, 2006; Triandis, 1994), especialmente, no que diz respeito à socialização organizacional de iniciantes, a qual tende a ser muito influenciada pela cultura por envolver metas e seleção ou avaliação de comportamentos e eventos (processos ou táticas) (Bauer \& Taylor, 2001). Desse modo, entende-se que abordar os efeitos das variáveis aqui enfocadas a partir de diferentes perspectivas culturais pode contribuir para distinguir os efeitos da resiliência e os efeitos da orientação cultural sobre os resultados da socialização organizacional, uma vez que os países envolvidos se caracterizam por orientações culturais pronunciadamente distintas (Hofstede \& Hofstede, 2005; Inglehart \& Baker, 2000; Inglehart \& Wezel, 2005).

Fundamentado neste propósito, o presente artigo se encontra estruturado por uma breve revisão de literatura acerca dos construtos de socialização organizacional e de resiliência, seguida da descrição das hipóteses estabelecidas, da metodologia adotada e da discussão dos resultados obtidos na condução da pesquisa. Encerra-se com a apresentação das considerações finais e indicações para estudos futuros no tema.

\section{Os Estudos em Socialização Organizacional}

A socialização organizacional tem sido abordada a partir de diferentes perspectivas. Alguns autores (Cooper-Thomas \& Anderson, 2006; Saks \& Ashforth, 1997) mencionam que são observáveis distintas divisões na literatura, com estudos voltados às táticas organizacionais, ao papel das diferenças individuais e da proatividade, aos conteúdos e à aprendizagem, além daqueles que integram diferentes enfoques na construção de modelos abrangentes que abordam as relações entre as variáveis imbricadas no processo. 
A discussão acerca do papel das diferenças individuais no processo de socialização organizacional teve início, quando as pesquisas começaram a demonstrar que os iniciantes reagem de modo distinto aos esforços organizacionais despendidos na promoção de sua adaptação e aprendizagem (Ashford \& Black, 1996; Reichers, 1987). Observou-se que muitas variáveis poderiam moderar, mediar ou predizer as relações entre as práticas de socialização e seus resultados, propiciando suporte à noção de que a efetividade dos programas de socialização dependeria, em parte, das características dos iniciantes (Saks \& Ashforth, 1997).

No intuito de se tornarem membros proficientes e se sentirem confortáveis na organização, tais iniciantes precisam aprender um conjunto de aspectos relacionados ao trabalho que são essenciais ao bom desempenho (Ashforth, Sluss, \& Saks, 2007; Lisbona, Morales, \& Palací, 2009). Visando identificar estas dimensões de conteúdo e aprendizagem que compõem o processo de socialização organizacional, alguns estudiosos têm sugerido diferentes tipologias (Chao, O’Leary-Kelly, Wolf, Klein, \& Gardner, 1994; Haueter, Macan, \& Winter, 2003; Taormina, 1997). Mais recentemente, Borges, Silva, Melo, Oliveira e Roazzi (2010) propuseram o Inventário de Socialização Organizacional (ISO), composto pela seguinte estrutura fatorial.

1. Acesso às Informações (políticas) - diz respeito ao conhecimento de datas importantes, processos organizacionais, critérios e poder.

2. Competência e Proatividade - refere-se à capacidade de criar, ser eficaz, produtivo, ativo, e de buscar informação.

3. Integração às Pessoas - envolve o sentimento de ser aceito pelos outros, incluído na equipe, na organização e participar nos processos de tomada de decisão.

4. Não-integração à Organização - inclui a ausência do domínio da linguagem, do emprego e do conhecimento acerca dos processos organizacionais e da cultura organizacional.

5. Qualificação Profissional - aborda o conhecimento e a experiência profissional, o domínio da linguagem profissional e das tarefas.

6. Objetivos e Valores Organizacionais - compreende o conhecimento e identificação com os objetivos e prioridades organizacionais e o conhecimento da história organizacional.

7. Linguagem e Tradição - abrange o domínio da linguagem profissional e organizacional, o conhecimento das tradições e a identificação das pessoas mais influentes.

Este inventário foi adotado no presente estudo para analisar os resultados de socialização organizacional obtidos pelos novos servidores aqui analisados. O fator de Não-integração à Organização foi invertido, sendo discutido em termos de Integração à Organização.

\section{A Resiliência e a Socialização Organizacional}

A resiliência tem sido definida na literatura como um fenômeno caracterizado por resultados positivos de adaptação na presença de ameaças ao desenvolvimento das pessoas (Masten, 2001), o qual possibilita a superação de situações de estresse e adversidades (Rutter, 1999). É por meio de comportamentos adaptados em resposta a situações de risco que ocorre a resiliência; de modo que é condição para analisar esse processo, a investigação de fatores de risco e de proteção em interação (Polleto \& Koller, 2006).

O que se denomina de fatores de risco são aquelas condições ou variáveis que são associadas com uma elevada possibilidade de ocorrência de resultados indesejados ou negativos, os quais envolvem comportamentos que podem comprometer a saúde, o bem-estar ou o desempenho social (Fraser, Richman, \& Galinsky, 1999; Jessor, Van Den Bos, Vanderryn, Costa, \& Turbin, 1995). Um 
evento estressor poderá representar condição de risco, dependendo da percepção, da interpretação e do sentido atribuído àquela vivência pelo indivíduo (Sanzovo \& Coelho, 2007).

Estando o estresse relacionado a alterações ambientais aversivas, perante as quais o indivíduo precisa apresentar respostas de adaptação, eventos que demandem algum tipo de ajustamento por parte do indivíduo podem ser considerados estressores. A este respeito, Vergara (2008) enfatiza os períodos de transição, em geral, como particularmente importantes para a resiliência, por acarretarem estresse considerável. No caso aqui enfocado, por exemplo, tem-se como fator gerador de estresse o período de transição, experimentado pelos novos servidores docentes e técnico-administrativos, após sua entrada na instituição, caracterizado como processo de socialização organizacional.

No que concerne aos fatores ou mecanismos de proteção, normalmente se lhes atribui a capacidade de facilitar o processo de superar o estresse e as adversidades. A característica que define tais fatores é a modificação da resposta de um individuo a uma situação de risco (Rutter, 1999). Vários são os recursos implicados nos mecanismos de proteção e as pesquisas levaram a delinear três amplas categorias (Jessor et al., 1995; Pinheiro, 2004): (a) características e recursos positivos do individuo; (b) condições familiares (boa qualidade das interações, coesão e apoio); e (c) sistemas de suporte externo (relações interpessoais extrafamiliares favoráveis).

Friborg, Hjemdal, Rosenvinge e Martinussen (2003) consideram que estas três categorias englobam os determinantes mais significativos de um ajustamento saudável ao estresse de longo prazo. Por essa razão, tomaram-nas como base para o desenvolvimento da Escala de Resiliência para Adultos (RSA), que inclui os recursos de proteção ali representados. Após introduzirem modificações e melhorias no instrumento, realizando sucessivos estudos de validação, os autores da RSA (Friborg, Barlaug, Martinussen, Rosenvinge, \& Hjemdal, 2005; Friborg, Hjemdal, Rosenvinge, \& Martinussen, 2003) chegaram a uma versão final da escala, composta de seis fatores: Percepção de si Mesmo, Futuro Planejado, Competência Social, Estilo Estruturado, Coesão Familiar e Recursos Sociais (Hjemdal et al., 2006). Tais fatores são descritos a seguir, à medida que se apresentam as hipóteses acerca das associações esperadas entre eles e os resultados de socialização organizacional (representados pelos sete fatores do Inventário de Socialização Organizacional [ISO]).

O primeiro fator da RSA, denominado Percepção de si mesmo, refere-se à confiança do indivíduo nas próprias capacidades, à expressão de autoeficácia e a uma visão positiva e realista acerca de si mesmo. Embora este fator ainda não tenha sido examinado nos estudos em socialização, associações positivas entre a autoeficácia e o aproveitamento de treinamentos (Saks, 1995), o comportamento proativo de busca de feedback e a construção de relacionamentos no trabalho (Gruman et al., 2006) têm sido apontadas nas pesquisas. Deste modo, espera-se que:

H1: O fator Percepção de si mesmo se mostrará como preditor dos fatores Acesso às Informações, Competência e Proatividade e Integração às Pessoas.

O segundo fator de resiliência, Futuro Planejado, se refere à medida na qual um indivíduo detém uma visão otimista do próprio futuro, a certeza de que pode ser bem-sucedido, e a habilidade de planejamento e estabelecimento de metas claras e alcançáveis. A noção expressa neste fator é de atuação no presente, visando a objetivos de médio e longo prazo. Como o alcance de bons resultados, em termos de desempenho organizacional e sucesso de longo prazo na carreira guarda estreita relação com o nível de socialização organizacional (Cooper-Thomas \& Anderson, 2006; Saks \& Asforth, 1997), entende-se que:

H2: O fator Futuro Planejado será preditor de todos os fatores de socialização organizacional.

Quanto ao fator Competência Social, este descreve a percepção do individuo a respeito de sua habilidade em iniciar contatos verbais e ser flexível em interações sociais, criar novas amizades e sentir-se à vontade em ambientes sociais. A ligação entre este fator e a integração às pessoas parece clara; mas, igualmente plausível, é a suposição de que a competência social esteja associada ao acesso às informações. Como suporte dessa proposição, uma pesquisa demonstrou relação positiva entre o fator Competência Social e a dimensão de personalidade denominada extroversão (Friborg et al., 
2005); a qual, por sua vez, se mostrou associada a elevados níveis de busca de informação e construção de relacionamentos (Wanberg \& Kammeyer-Mueller, 2000). Ainda, como o fator de socialização referente ao conhecimento da Linguagem e Tradição, abrange o domínio da linguagem profissional e organizacional, das tradições e a identificação das pessoas mais influentes no meio, entende-se que a competência social também se encontre a ele associada. A terceira hipótese, portanto, é de que:

H3: O fator Competência Social se mostrará como preditor dos fatores Acesso às Informações, Integração às Pessoas e Linguagem e Tradição.

Tomando-se o fator de resiliência Estilo Estruturado, que envolve a capacidade dos indivíduos de organizar o próprio tempo, estabelecendo objetivos e prazos e mantendo regras e rotinas na vida diária, tem-se que os indivíduos com escores elevados neste fator tendem a ser organizados, responsáveis e conservadores. Assim, espera-se que o mesmo se associe ao Acesso às Informações, Qualificação Profissional, Integração à Organização e conhecimento da Linguagem e Tradição e dos Objetivos e Valores Organizacionais. Dado, contudo, que as características do fator Estilo Estruturado tendem a estar presentes em indivíduos com elevado senso de responsabilidade (Friborg et al., 2005) e pesquisa anterior aponta a não identificação de relações entre tal dimensão de personalidade e a busca por informação e feedback (Wanberg \& Kammeyer-Mueller, 2000), propõe-se a seguinte hipótese:

H4: O fator Estilo Estruturado se mostrará como preditor dos fatores de Qualificação Profissional; Integração à Organização; Objetivos e Valores Organizacionais; e Linguagem e Tradição.

Indivíduos com elevados escores no fator de resiliência denominado Coesão Familiar são aqueles que experimentam relações de boa qualidade com a família, em termos de comunhão de valores e visão de futuro, união, lealdade e simpatia mútua. Em outras palavras, estes indivíduos têm nos seus familiares um referencial de segurança e uma fonte de apoio para fazer face aos desafios com que se defrontam em suas vidas.

No que concerne ao processo de socialização organizacional, entretanto, pesquisas apontam que a utilização do apoio de familiares como referência, além de gerar impactos negativos (Louis, 1980; Settoon \& Adkins, 1997), só exerce influência nos resultados do processo durante o período de entrada na organização. Estudo conduzido por Settoon e Adkins (1997) demonstrou que, após o período de seis meses, nenhuma relação foi verificada entre o apoio de familiares e os resultados de socialização organizacional, uma vez que, no decorrer de tal período, os iniciantes tiveram a oportunidade de construir relações e estabelecer fontes de referência intraorganizacionais. Considerando-se, portanto, que na presente pesquisa os dados relativos aos novos servidores foram coletados quando os respondentes já contavam, no mínimo, 18 meses na organização, espera-se que:

H5: O fator de Coesão Familiar não irá predizer nenhum dos fatores de socialização organizacional.

Por fim, o fator de Recursos Sociais trata do suporte social oferecido por pessoas que não fazem parte do núcleo familiar (colegas de trabalho, por exemplo), e que propicia o sentimento de coesão, a simpatia, o encorajamento e a ajuda em situações difíceis. É comum identificar a influência positiva do apoio de colegas no trabalho em relação aos resultados da socialização organizacional. Conforme constatado por Settoon e Adkins (1997), a utilização deste tipo de apoio relacionou-se negativamente com o conflito de papel e com as intenções de deixar a organização. Bravo, Peiró, Rodriguez e Whitely (2003) observaram que a boa qualidade das relações sociais na organização tende a reduzir o estresse e encorajar a proatividade dos iniciantes. Reichers (1987), por sua vez, já apontava que o estabelecimento da identidade situacional e a construção de sentido a respeito de eventos, práticas e procedimentos organizacionais tendem a se realizar mais rapidamente, quando os iniciantes interagem com outros membros da organização. Apresenta-se, então, a hipótese de que: 
H6: O fator de Recursos Sociais será preditor dos fatores de Acesso às Informações; Integração às Pessoas; Integração à Organização; Objetivos e Valores Organizacionais; e, Linguagem e Tradição.

Por fim, como o estudo envolve novos servidores brasileiros e noruegueses, é importante refletir em que o papel da resiliência em relação à socialização organizacional pode variar nestes diferentes contextos culturais. Desta forma, a última hipótese do estudo é de que:

H7: A capacidade preditiva da resiliência em relação à socialização organizacional irá diferir entre os novos servidores da universidade brasileira e da universidade norueguesa.

\section{O Método}

\section{Participantes do estudo}

$\mathrm{Na}$ constituição da população encontram-se os novos servidores docentes e técnicoadministrativos das duas universidades públicas (brasileira e norueguesa). A amostra foi constituída de 135 novos servidores, sendo 72 deles brasileiros e 63 noruegueses. Trata-se de uma amostra acidental, uma vez que o critério adotado na sua constituição foi de acessibilidade dos participantes (Kish, 1996; Scarparo, 2000).

No que concerne ao perfil dos respondentes, os novos servidores têm idade entre 30 e 60 anos (86,2\% dos brasileiros e 93,7\% dos noruegueses), sendo a maioria deles funcionários técnicoadministrativos $(54,2 \%$ dos brasileiros e $52,4 \%$ dos noruegueses) e do sexo feminino $(61,1 \%$ dos brasileiros e 52,4\% dos noruegueses). O tempo de serviço na universidade foi de 24 a 69 meses para os brasileiros $(\mathrm{M}=52,9$ meses; $\mathrm{SD}=10,8)$ e de 18 a 70 meses para os noruegueses $(\mathrm{M}=44,6$ meses; $\mathrm{SD}=13,6)$.

\section{Instrumentos da pesquisa}

Para a obtenção dos dados referentes à socialização organizacional dos servidores foi utilizado o Inventário de Socialização Organizacional (ISO) (Borges, Silva, Melo, Oliveira, \& Roazzi, 2010). O inventário conta com 45 itens, aos quais os participantes respondem segundo uma escala que varia entre -2 (forte discordância) e 2 (forte concordância), passando por -1 (discordância), 0 (neutro) e 1 (concordância). O ISO foi submetido à análise fatorial, mostrando-se capaz de mensurar sete fatores (anteriormente mencionados), os quais apresentam aceitável consistência (alfas de Cronbach variando de 0,70 a 0,83$)$.

Para a aplicação entre os novos servidores na Noruega, o ISO foi traduzido, com o auxílio de pesquisadores multilíngues, para o norueguês. Tal procedimento se encontra em conformidade com a recomendação de Ros e Gouveia (2006), que apontam como mais adequado nesses casos: a consulta a um grupo de especialistas nos idiomas necessários ao estudo ou a tradução de uma língua para outra, seguida da tradução reversa (backtranslation).

Quanto às informações concernentes à resiliência dos novos servidores, foram obtidas por meio da aplicação de questionário estruturado, denominado Escala de Resiliência para Adultos (RSA) (Hjemdal et al., 2006), que tem sua versão original em norueguês e foi adaptada e validada para a língua portuguesa por Hjemdal, Roazzi, Dias, Roazzi e Vikan (2009). A RSA contém 33 itens, estruturados em uma escala de sete pontos em formato de diferencial semântico, na qual cada item é organizado como um continuum, cujos opostos apresentam alternativas de resposta com conteúdo positivo e negativo. Após o processo de adaptação e validação, a estrutura fatorial foi confirmada, mostrando-se capaz de mensurar seis fatores (anteriormente mencionados), cujos alfas de Cronbach variam de 0,56 a 0,79 . 


\section{Coleta de dados}

Todos os participantes da pesquisa receberam um envelope com um questionário de socialização organizacional, um questionário de resiliência e uma ficha sociodemográfica para a identificação do perfil dos respondentes. Os procedimentos de coleta ocorreram em duas fases, envolvendo os dois diferentes grupos constituintes da população do estudo.

A primeira delas foi realizada no ano de 2007, junto a 153 novos servidores brasileiros, enviando-se correspondência interna aos departamentos, por meio de malote institucional. Estes servidores receberam os questionários com instruções de preenchimento, um envelope-resposta e uma carta em que se esclarece os motivos da pesquisa e se solicita a contribuição dos respondentes. $\mathrm{Na}$ segunda fase da coleta, realizada no ano de 2008, envolvendo 154 novos servidores noruegueses, foi empregada a mesma estratégia utilizada na fase anterior. No final, foi contabilizada a devolução de 72 questionários válidos na universidade brasileira (47\% de retorno) e 63 na universidade norueguesa (retorno de 40,9\%).

\section{Tratamento dos dados}

Análises de regressão hierárquica (método enter) foram realizadas; as primeiras envolveram todos os participantes $(\mathrm{N}=135)^{(1)}$, no intuito de examinar a capacidade explicativa da resiliência com relação aos resultados da socialização organizacional, independentemente da nacionalidade e da ocupação. Cada fator de socialização organizacional foi considerado como variável critério, em cada uma das análises. Como variáveis de controle foram incluídas, no primeiro bloco a nacionalidade e a ocupação e, no segundo bloco, como variáveis antecedentes, os fatores de resiliência.

As demais análises foram conduzidas separadamente para os novos servidores brasileiros $(\mathrm{N}=72)$ e noruegueses $(\mathrm{N}=63)$, com vistas a observar se a capacidade explicativa da resiliência com relação aos resultados da socialização, além dos efeitos da ocupação, iria diferir entre os participantes dos dois países. Nestas, embora cada um dos fatores de socialização organizacional continuasse como variável critério, em cada uma das análises, apenas a ocupação entrou como variável de controle no primeiro bloco e um escore total de resiliência (variável antecedente) foi incluído no segundo bloco.

A opção por calcular e utilizar um escore médio total para a RSA foi feita seguindo os procedimentos recomendados por Tabachnick e Fidell (2007), que alertam para a importância de observar a proporção entre o número de participantes e o de variáveis antecedentes e de controle empregadas nas análises, para evitar resultados muito otimistas e/ou equivocados. Como o total de participantes das universidades brasileira e norueguesa foi de 72 e 63 indivíduos, respectivamente, o conjunto de variáveis antecedentes e de controle, para cada grupo, poderia ser de duas, no máximo.

\section{Análise dos Resultados}

Os resultados das análises descritivas e de correlação encontram-se dispostos na Tabela 1. Como é possível notar, a variável nacionalidade correlacionou-se de forma significativa e moderada com os fatores de socialização que se referem à Integração às Pessoas $(r=-0,22)$ e à Integração à Organização $(r=0,19)$ e com o fator de resiliência Competência Social $(r=0,33)$. Verifica-se, portanto, uma tendência de melhor integração social entre os servidores da universidade norueguesa e de melhor integração organizacional e competência social entre os servidores da universidade brasileira.

Quanto à ocupação, observam-se correlações negativas e moderadas com dois dos fatores de socialização (Competência e Proatividade e Objetivos e Valores Organizacionais) e três dos fatores de resiliência (coeficientes de Pearson entre -0,18 e -0,24). Tal resultado é indicativo de que os servidores docentes apresentaram tendência de melhor socialização e resiliência nos referidos fatores. 
Os valores das correlações entre os fatores de socialização variaram de 0,25 a 0,68 e entre os de resiliência, de 0,21 a 0,56 , o que denota certa sobreposição nos conteúdos expressos nestes fatores, expressando, em parte, a complexidade de tais fenômenos, cujas dimensões não são exatamente excludentes. No que tange à utilização de escores em fatores correlacionados entre si como variáveis antecedentes, autores como Tabachnick e Fidell (2007) afirmam que a análise de regressão apoia tal característica. Importa ainda esclarecer que, embora haja correlações, não há multicolinearidade (altos coeficientes de correlação entre as variáveis, como $r>0,70$ ), o que denota suficiente distinção entre os fatores.

Tomando-se as correlações entre os fatores de socialização organizacional e de resiliência, notase, de modo geral, a tendência a associações elevadas entre ambos, de forma que o coeficiente menos expressivo $(r=0,17)$ se deu entre os fatores Linguagem e Tradição e Competência Social e o mais expressivo $(r=0,52)$ entre os fatores de Acesso às Informações e Futuro Planejado.

Visando investigar se os fatores de resiliência contribuem para explicar a variância nos resultados de socialização organizacional, além das diferenças de nacionalidade e ocupação, foram realizadas análises de regressão hierárquica. Os resultados obtidos (Tabela 2) demonstram que as variáveis de controle, do primeiro bloco, explicam significativamente a variância dos fatores de Competência e Proatividade $\left(R^{2}=0,06\right)$, Integração às Pessoas $\left(R^{2}=0,07\right)$, Integração à Organização $\left(R^{2}=0,06\right)$ e Objetivos e Valores Organizacionais $\left(R^{2}=0,09\right)$. 
Tabela 1

Médias, Desvio Padrão e Coeficientes de Correlação para as Variáveis do Estudo

\begin{tabular}{|c|c|c|c|c|c|c|c|c|c|c|c|c|c|c|c|c|c|}
\hline Variáveis & $\mathbf{M}$ & DP & 1 & 2 & 3 & 4 & 5 & 6 & 7 & 8 & 9 & 10 & 11 & 12 & 13 & 14 & 15 \\
\hline 1. Nacionalidade & - & - & - & & & & & & & & & & & & & & \\
\hline 2. Ocupação & - & - & 0,02 & - & & & & & & & & & & & & & \\
\hline 3. Acesso às Informações & 3,76 & 0,75 & $-0,12$ & $-0,15$ & - & & & & & & & & & & & & \\
\hline $\begin{array}{l}\text { 4. Competência e Pró- } \\
\text { atividade }\end{array}$ & 4,35 & 0,43 & 0,04 & $-0,24^{*}$ & $0,61 *$ & - & & & & & & & & & & & \\
\hline 5. Integração às Pessoas & 4,13 & 0,79 & $-0,22 *$ & $-0,14$ & $0,57 * *$ & $0,39 * *$ & - & & & & & & & & & & \\
\hline $\begin{array}{l}\text { 6. Integração à } \\
\text { Organização }\end{array}$ & 4,25 & 0,59 & $0,19 *$ & $-0,15$ & $0,55^{* *}$ & $0,50 * *$ & $0,47 * *$ & - & & & & & & & & & \\
\hline $\begin{array}{l}\text { 7. Qualificação } \\
\text { Profissional }\end{array}$ & 4,48 & 0,51 & 0,00 & $-0,14$ & $0,35^{* *}$ & $0,57 * *$ & $0,33 * *$ & $0,25^{* *}$ & - & & & & & & & & \\
\hline $\begin{array}{l}\text { 8. Objetivos e Valores } \\
\text { Organizacionais }\end{array}$ & 3,89 & 0,67 & $-0,09$ & $-0,29 *$ & $0,68 * *$ & $0,50 * *$ & $0,66^{* *}$ & $0,56^{*}$ & $0,36 * *$ & - & & & & & & & \\
\hline 9. Linguagem e Tradição & 3,89 & 0,61 & $-0,14$ & $-0,09$ & $0,68 * *$ & $0,40 * *$ & $0,48 * *$ & $0,49 * *$ & $0,32 * *$ & $0,68 * *$ & - & & & & & & \\
\hline $\begin{array}{l}\text { 10. Percepção de si } \\
\text { Mesmo }\end{array}$ & 5,49 & 0,02 & 0,14 & $-0,23 *$ & $0,44 * *$ & $0,40 * *$ & $0,34 * *$ & $0,32 * *$ & $0,23 * *$ & $0,41 * *$ & $0,32 * *$ & - & & & & & \\
\hline 11. Futuro Planejado & 5,72 & 0,11 & 0,09 & $-0,24 *$ & $0,52 * *$ & $0,42 * *$ & $0,50 * *$ & $0,45^{* *}$ & $0,30 * *$ & $0,47 * *$ & $0,42 * *$ & $0,56 * *$ & - & & & & \\
\hline 12. Competência Social & 5,42 & 0,06 & $0,33 *$ & $-0,18^{*}$ & 0,21 & $0,29 * *$ & $0,28 * *$ & $0,23 * *$ & $0,26 * *$ & $0,24 * *$ & $0,17 *$ & $0,55 * *$ & $0,42 * *$ & - & & & \\
\hline 13. Estilo Estruturado & 5,43 & 0,03 & 0,04 & $-0,09$ & $0,34 * *$ & $0,26 * *$ & $0,19 *$ & $0,19 *$ & $0,18^{*}$ & $0,33 * *$ & $0,36 * *$ & $0,35 * *$ & $0,42 * *$ & $0,25 * *$ & - & & \\
\hline 14. Coesão Familiar & 5,69 & 0,05 & 0,12 & $-0,09$ & 0,03 & 0,16 & $0,23 * *$ & $0,20 *$ & 0,14 & 0,12 & 0,04 & $0,41 * *$ & $0,23 * *$ & $0,40 * *$ & $0,21^{*}$ & - & \\
\hline 15. Recursos Sociais & 6,08 & 0,75 & 0,09 & $-0,14$ & $0,33 * *$ & $0,33 * *$ & $0,44 * *$ & $0,29 * *$ & $0,37 * *$ & $0,33 * *$ & $0,29 * *$ & $0,46^{* *}$ & $0,41 * *$ & $0,55 * *$ & $0,24 * *$ & $0,54 * *$ & - \\
\hline
\end{tabular}

Nota. $N=135 / * \mathrm{p}<0,05 \quad * * \mathrm{p}<0,01$.

Nacionalidade: 1 = norueguesa e 2 = brasileira / Ocupação: 1 = servidores docentes e $2=$ servidores técnico-administrativos. 
Tabela 2

Preditores dos Fatores de Socialização Organizacional

\begin{tabular}{|c|c|c|c|c|c|c|c|c|c|c|c|c|c|c|}
\hline \multirow[t]{2}{*}{ Preditores } & \multicolumn{2}{|c|}{$\begin{array}{c}\text { Acesso às } \\
\text { Informações } \beta\end{array}$} & \multicolumn{2}{|c|}{$\begin{array}{l}\text { Competência e } \\
\text { Pró-atividade } \beta\end{array}$} & \multicolumn{2}{|c|}{$\begin{array}{c}\text { Integração às } \\
\text { Pessoas } \beta\end{array}$} & \multicolumn{2}{|c|}{$\begin{array}{c}\text { Integração à } \\
\text { Organização } \beta\end{array}$} & \multicolumn{2}{|c|}{$\begin{array}{c}\text { Qualificação } \\
\text { Profissional } \beta\end{array}$} & \multicolumn{2}{|c|}{$\begin{array}{c}\text { Objetivos e } \\
\text { Valores } \\
\text { Organizacionais } \beta\end{array}$} & \multicolumn{2}{|c|}{$\begin{array}{c}\text { Linguagem e } \\
\text { Tradição } \beta\end{array}$} \\
\hline & Bloco 1 & Bloco 2 & Bloco 1 & Bloco 2 & Bloco 1 & Bloco 2 & Bloco 1 & Bloco 2 & Bloco 1 & Bloco 2 & Bloco 1 & Bloco 2 & Bloco 1 & Bloco 2 \\
\hline \multicolumn{15}{|c|}{ Variáveis de controle ( Bloco 1) } \\
\hline Nacionalidade & $-0,12$ & $-0,15^{*}$ & 0,04 & $-0,01$ & $-0,22$ & $-0,30 * *$ & $0,19 *$ & 0,17 & 0,00 & $-0,05$ & $-0,09$ & $-0,13$ & $-0,14$ & $-0,17 *$ \\
\hline Ocupação & $-0,15$ & 0,00 & $-0,24 * *$ & $-0,12$ & $-0,13$ & 0,01 & $-0,15$ & $-0,05$ & $-0,14$ & $-0,06$ & $-0,29 * *$ & $-0,16$ & $-0,08$ & 0,03 \\
\hline \multicolumn{15}{|c|}{ Variáveis Antecedentes do Bloco 2} \\
\hline Percepção si Mesmo & & $0,29 * *$ & & 0,19 & & 0,03 & & 0,05 & & $-0,01$ & & 0,19 & & 0,16 \\
\hline Futuro Planejado & & $0,31 * *$ & & $0,19 *$ & & $0,40 * *$ & & $0,38 * *$ & & 0,14 & & $0,24 *$ & & $0,24 *$ \\
\hline Competência Social & & $-0,09$ & & 0,00 & & 0,06 & & $-0,11$ & & 0,06 & & $-0,04$ & & $-0,05$ \\
\hline Estilo Estruturado & & 0,14 & & 0,08 & & $-0,05$ & & $-0,01$ & & 0,05 & & 0,14 & & $0,21 *$ \\
\hline Coesão Familiar & & $-0,27 * *$ & & $-0,09$ & & $-0,00$ & & 0,05 & & $-0,09$ & & $-0,13$ & & $-0,20 *$ \\
\hline Recursos Sociais & & $0,25 * *$ & & 0,17 & & $0,26 * *$ & & 0,12 & & $0,31 * *$ & & $0,19 *$ & & $0,22 *$ \\
\hline $\mathrm{R}$ & 0,20 & $0,64 * *$ & $0,24 * *$ & $0,50 * *$ & $0,26 *$ & $0,63 * *$ & $0,25^{*}$ & $0,50 * *$ & 0,14 & $0,42 * *$ & $0,30 * *$ & $0,58 * *$ & 0,16 & $0,54 * *$ \\
\hline $\mathrm{R}^{2}$ & 0,04 & $0,41 * *$ & $0,06 * *$ & $0,25^{* *}$ & $0,07 *$ & $0,40 * *$ & $0,06 *$ & $0,25 * *$ & 0,02 & $0,18^{* *}$ & $0,09 * *$ & $0,34 * *$ & 0,03 & $0,30 * *$ \\
\hline Mudança em $\mathrm{R}^{2}$ & & $0,37 * *$ & & $0,19 * *$ & & $0,33 * *$ & & $0,19 * *$ & & $0,16^{* *}$ & & $0,25 * *$ & & $0,27 * *$ \\
\hline
\end{tabular}

Nota. $N=135 / * \mathrm{p}<0,05 \quad * * \mathrm{p}<0,01$

Nacionalidade: 1 = norueguesa e 2 = brasileira / Ocupação: $1=$ servidores docentes e $2=$ servidores técnico-administrativos. 
Após a entrada dos fatores de resiliência, no segundo bloco, uma explicação significativa foi adicionada à variância de todos os fatores de socialização organizacional, com mudanças em $\mathrm{R}^{2}$ oscilando entre 16\% (para o fator de Qualificação Profissional) e 37\% (para o fator de Acesso às Informações), o que indica que a resiliência oferece uma explicação incremental considerável, além da nacionalidade e da ocupação.

Examinando a contribuição preditiva de cada um dos fatores de resiliência, em particular, constatou-se uma associação positiva entre o fator Percepção de si mesmo e o fator de socialização organizacional referente ao Acesso às Informações $(\beta=0,29)$. Tal resultado corrobora parcialmente a hipótese 1, uma vez que esta previa o fator Percepção de si mesmo em relação positiva com os fatores de Acesso às Informações, Competência e Proatividade e Integração às Pessoas.

Considerando-se que a Percepção de si mesmo se refere à autoconfiança e à autoeficácia, sua predição do Acesso às Informações acerca de processos organizacionais, critérios e poder é, de certa forma, consistente com achados anteriores sobre o papel da autoeficácia para o aproveitamento de treinamentos (Saks, 1995); e para o comportamento proativo de busca de feedback e de construção de relacionamentos no trabalho (Gruman et al., 2006).

No que diz respeito ao fator de resiliência Futuro Planejado, nota-se que este foi preditor significativo de quase todos os fatores de socialização organizacional (coeficientes $\beta$ entre 0,19 e $0,40)$, exceto aquele de Qualificação Profissional. Sua capacidade de predição foi maior em relação à Integração às Pessoas $(\beta=0,40)$ e menor no que se refere à Competência e Proatividade $(\beta=0,19)$. Tem-se, dessa forma, um suporte parcial à hipótese 2 , a qual previa a predição de todos os fatores de socialização organizacional pelo fator de resiliência Futuro Planejado.

Observa-se, portanto, que os iniciantes com uma orientação mais positiva em relação ao futuro e com maior habilidade de planejamento e estabelecimento de metas tendem a distinguir, de forma mais clara, as alternativas de ação que conduzem ao alcance de um bom desempenho na organização e ao sucesso de longo prazo na carreira; isso envolve, conforme estudiosos do tema (Saks \& Asforth, 1997; Cooper-Thomas \& Anderson, 2006), a necessidade de se socializar satisfatoriamente no ambiente organizacional. Assim, entende-se que a efetividade dos programas de socialização pode residir, em parte, na sua capacidade de fortalecer as habilidades dos iniciantes em planejar e formular metas claras e alcançáveis e, ainda, as oportunidades que as organizações concedem aos iniciantes para realizar tais metas.

Reportando-se ao fator de Competência Social, observa-se que este foi o único que não se mostrou relacionado a nenhum dos fatores de socialização organizacional. Como consequência, a hipótese 3 foi refutada, uma vez que previa uma relação positiva entre o fator de Competência Social e os fatores de socialização que descrevem o Acesso às Informações, a Integração às Pessoas e o conhecimento da Linguagem e Tradição.

No que tange ao papel desempenhado pelo fator de resiliência Estilo Estruturado, os resultados apontaram uma associação positiva deste com o fator de socialização que aborda o conhecimento da Linguagem e Tradição $(\beta=0,21)$. Retomando a hipótese 4 , que previa uma associação do fator de Estilo Estruturado com os fatores de Qualificação Profissional, Integração à Organização, Objetivos e Valores Organizacionais, e Linguagem e Tradição, tira-se por conclusão que esta foi apenas parcialmente corroborada.

A predição verificada somente em relação ao fator de Linguagem e Tradição pode dever-se ao fato de que este, por envolver o domínio da linguagem profissional e institucional, o conhecimento das tradições e das pessoas mais influentes, venha a ser especialmente relevante para os indivíduos com escores elevados no fator Estilo Estruturado. Tais indivíduos, na busca de organizar de forma adequada o próprio tempo, estabelecem objetivos e prazos e preferem manter regras e rotinas fixas; precisam, para isso, compreender como funcionam normalmente os procedimentos no seu novo ambiente de trabalho, de maneira que possam adequá-los à sua rotina, ao mesmo tempo que buscam adequá-la a si. Dessa forma, os iniciantes que tendem a apresentar um estilo estruturado podem 
perceber o conhecimento da linguagem e tradição organizacionais como aspecto crítico, para que possam sentir-se mais confortáveis no seu processo de adaptação ao ambiente organizacional.

Atuando em direção oposta àquela identificada quanto ao fator Estilo Estruturado, a Coesão Familiar aparece como fator de resiliência que dificulta o conhecimento da Linguagem e Tradição ( $\beta=-$ $0,20)$, além de prejudicar o Acesso às Informações $(\beta=-0,27)$. Tais resultados fazem com que seja refutada a hipótese 5, segundo a qual o fator de Coesão Familiar não se mostraria relacionado a nenhum dos fatores de socialização organizacional.

Tem-se aqui um achado interessante, uma vez que a hipótese 5 foi estabelecida com base em estudos anteriores (Louis, 1980; Settoon \& Adkins, 1997), os quais discutiam que os impactos negativos da utilização do apoio de familiares, durante a socialização organizacional, ocorriam somente nos seis primeiros meses na organização. O que aqui se observou, todavia, foi que os resultados obtidos contradizem, em parte, os achados daqueles autores, porque, a despeito da confirmação dos efeitos negativos do apoio familiar na socialização organizacional, esta se verificou entre servidores com tempo superior a seis meses de serviço na instituição.

Quanto às relações negativas identificadas, cumpre considerar que o iniciante, ao encontrar apoio entre familiares, pode não esforçar-se por construir relações de cooperação no trabalho. Nestas circunstâncias, entretanto, embora o apoio familiar possa ser efetivo para proporcionar conforto e amenizar o estresse, não tenderá a ser para a aquisição de informações organizacionais, mais prováveis de ser obtidas junto aos colegas de trabalho. Os resultados aqui obtidos reforçam essa concepção, uma vez que os fatores de socialização influenciados negativamente pela coesão familiar se encontram estreitamente correlacionados $(\mathrm{r}=0,68)$. O que parece ocorrer, portanto, é uma reação em cadeia, na qual os novos servidores com maior coesão familiar têm menor Acesso às Informações no trabalho, o que gera dificuldades para o conhecimento da Linguagem e Tradição organizacionais.

Se os resultados concernentes à Coesão Familiar contradizem parcialmente os achados de Settoon e Adkins (1997), o mesmo não ocorre, todavia, para o fator de Recursos Sociais, uma vez que este se mostrou positivamente relacionado a vários fatores de socialização organizacional: Acesso às Informações $(\beta=0,25)$, Integração às Pessoas $(\beta=0,26)$, Qualificação Profissional $(\beta=0,31)$, Objetivos e Valores Organizacionais $(\beta=0,19)$ e Linguagem e Tradição $(\beta=0,22)$. Como a hipótese 6 previa relações positivas do fator de Recursos Sociais com os fatores de Acesso às Informações, Competência e Proatividade, Integração às Pessoas, Integração à Organização, Objetivos e Valores Organizacionais e Linguagem e Tradição, constata-se que ela obteve suporte parcial.

As associações previstas e observadas entre o fator de Recursos Sociais e os fatores de socialização organizacional, acima descritos, corroboram a noção de que o suporte social constitui uma influência positiva nos resultados da socialização, uma vez que contribui para que os iniciantes possam administrar as demandas típicas do período de ajustamento (Bravo, Peiró, Rodriguez, \& Whitely, 2003; Reichers, 1987; Settoon \& Adkins, 1997). Nos casos aqui analisados, os iniciantes que contaram com tal suporte relataram sentir-se mais integrados aos colegas e informados acerca dos critérios, processos, objetivos, valores, linguagem e tradição organizacionais.

Ainda, a associação positiva não esperada entre os fatores de Recursos Sociais e Qualificação Profissional é indicativa de que o primeiro, por fortalecer a rede de contatos no trabalho, confere maiores oportunidades de aprendizagem dos aspectos relevantes à atuação profissional, aumentando o domínio da tarefa e a percepção de qualificação para ela.

Tomando, por sua vez, a contribuição das variáveis de nacionalidade e ocupação, predições significativas são também observadas. A nacionalidade, por exemplo, aparece como preditora dos seguintes fatores de socialização: Acesso às Informações $(\beta=-0,15$ / bloco 2$)$, Integração às Pessoas ( $\beta=-0,30$ / bloco 2), Integração à Organização $(\beta=0,19$ / bloco 1$)$ e Linguagem e Tradição $(\beta=-0,17$ / bloco 2). Tais resultados indicam que os novos servidores da universidade norueguesa tenderam a obter maior Acesso às Informações, Integração às Pessoas e Conhecimento da Linguagem e da 
Tradição organizacionais; ao passo que os novos servidores da universidade brasileira relataram maior Integração à Organização.

No que concerne à ocupação, esta se mostrou preditora apenas dos fatores Competência e Proatividade ( $\beta=-0,24$ / bloco 1$)$ e Objetivos e Valores Organizacionais $(\beta=-0,29$ / bloco 1$)$. Tais achados são indicativos de que o tipo de ocupação impactou os resultados da socialização: os docentes obtiveram melhores resultados nos aspectos acima descritos, comparativamente aos funcionários técnico-administrativos.

Por fim, com vistas a comparar a relação entre a resiliência e a socialização organizacional entre os servidores das duas universidades analisadas, novas análises de regressão hierárquica foram realizadas (Tabela 3 ).

Tabela 3

Análise de Regressão Hierárquica - Novos Servidores Brasileiros e Noruegueses

\begin{tabular}{|c|c|c|c|c|}
\hline \multirow[t]{2}{*}{ Variáveis } & \multicolumn{2}{|c|}{ Brasileiros $(\mathrm{N}=72)$} & \multicolumn{2}{|c|}{ Noruegueses $(\mathrm{N}=63)$} \\
\hline & $\mathbf{R}^{2}$ & Mud. $\mathbf{R}^{2}$ & $\mathbf{R}^{2}$ & Mud. $\mathbf{R}^{2}$ \\
\hline \multicolumn{5}{|l|}{ Variável Critério - Acesso às Informações } \\
\hline Variável de controle (Bloco 1) - Ocupação & $0,13 * *$ & & 0,02 & \\
\hline Variável antecedente (Bloco 2) - Resiliência & $0,29 * *$ & $0,16 * *$ & $0,17 * *$ & $0,15^{* *}$ \\
\hline \multicolumn{5}{|c|}{ Variável Critério - Competência e Pró-atividade } \\
\hline Variável de controle (Bloco 1) - Ocupação & $0,13 * *$ & & 0,01 & \\
\hline Variável antecedente (Bloco 2) - Resiliência & $0,32 * *$ & $0,19 * *$ & 0,07 & 0,06 \\
\hline \multicolumn{5}{|l|}{ Variável Critério - Integração às Pessoas } \\
\hline Variável de controle (Bloco 1) - Ocupação & $0,05^{*}$ & & 0,00 & \\
\hline Variável antecedente (Bloco 2) - Resiliência & $0,32 * *$ & $0,27 * *$ & $0,20 * *$ & $0,20 * *$ \\
\hline \multicolumn{5}{|l|}{ Variável Critério - Integração à Organização } \\
\hline Variável de controle (Bloco 1) - Ocupação & $0,08^{*}$ & & 0,00 & \\
\hline Variável antecedente (Bloco 2) - Resiliência & $0,14 * *$ & $0,06 * *$ & $0,17 * *$ & $0,17 * *$ \\
\hline \multicolumn{5}{|l|}{ Variável Critério - Qualificação Profissional } \\
\hline Variável de controle (Bloco 1) - Ocupação & 0,01 & & 0,05 & \\
\hline Variável antecedente (Bloco 2) - Resiliência & $0,16^{* *}$ & $0,15^{* *}$ & 0,09 & 0,04 \\
\hline \multicolumn{5}{|c|}{ Variável Critério - Objetivos e Valores Organizacionais } \\
\hline Variável de controle (Bloco 1) - Ocupação & $0,21 * *$ & & 0,00 & \\
\hline Variável antecedente (Bloco 2) - Resiliência & $0,39 * *$ & $0,18 * *$ & 0,08 & 0,08 \\
\hline \multicolumn{5}{|l|}{ Variável Critério - Linguagem e Tradição } \\
\hline Variável de controle (Bloco 1) - Ocupação & 0,05 & & 0,01 & \\
\hline Variável antecedente (Bloco 2) - Resiliência & $0,21 * *$ & $0,16 * *$ & $0,10^{*}$ & $0,09 *$ \\
\hline
\end{tabular}

Nota. Mud. $\mathrm{R}^{2}=$ Mudança em $\mathrm{R}^{2}$.

$* \mathrm{p}<0,05 \quad * * \mathrm{p}<0,01$.

Os achados apontam somente entre os brasileiros a ocupação explicou significativamente a variância dos fatores de socialização, a saber: Acesso às Informações $\left(\mathrm{R}^{2}=0,13\right)$; Competência e Proatividade $\left(R^{2}=0,13\right)$; Integração às Pessoas $\left(R^{2}=0,05\right)$; Integração à Organização $\left(R^{2}=0,08\right)$ e 
Objetivos e Valores Organizacionais $\left(\mathrm{R}^{2}=0,21\right)$; o escore total de resiliência adicionou uma explicação incremental significativa, além da ocupação, à variância de todos os fatores de socialização: mudanças em $\mathrm{R}^{2}$ entre $6 \%$ e $27 \%$.

Entre os noruegueses, a resiliência explicou a variância de apenas quatro dos fatores de socialização organizacional, com uma predição adicionada de $15 \%$ para o Acesso às Informações, $20 \%$ para a Integração às Pessoas, $17 \%$ para a Integração à Organização e $9 \%$ para a Linguagem e Tradição. Observa-se, ainda, que a parcela da variância explicada pela resiliência, entre estes servidores, tendeu a ser menor do que a identificada entre os novos servidores da universidade brasileira. Tais achados corroboram a hipótese 7 , de que a capacidade preditiva da resiliência em relação à socialização organizacional iria diferir entre os novos servidores das universidades brasileira e norueguesa.

\section{Considerações Finais}

O presente estudo contribuiu para ampliar a compreensão acerca do papel das diferenças individuais no processo de adaptação a um novo cargo e a uma nova realidade organizacional, demonstrando a relevância da resiliência para a experiência de socialização. Os resultados demonstraram, de modo geral, que os fatores de resiliência contribuíram significativamente para explicar todos os resultados de socialização aqui analisados, independentemente da nacionalidade e da ocupação. Mais especificamente, a contribuição preditiva dos fatores de resiliência Percepção de si mesmo, Futuro Planejado, Estilo Estruturado, Coesão Familiar e Recursos sociais se fizeram notar em diferentes aspectos.

Destacam-se, entre estes, os resultados verificados para os fatores Futuro Planejado e Recursos Sociais. O primeiro permitiu verificar a importância das habilidades de planejamento e estabelecimento de metas para o sucesso em variados aspectos da socialização organizacional, o que sugere uma via de ação para a estruturação de programas de socialização que estimulem os iniciantes à definição de objetivos, buscando interfaces possíveis entre aspirações pessoais e expectativas e oportunidades organizacionais. O segundo, isto é, o fator de Recursos Sociais, confirmou-se como fonte de apoio ao iniciante, reforçando resultados de pesquisas anteriores (Bravo et al., 2003; Settoon \& Adkins, 1997) acerca de sua influência para uma melhor integração aos colegas, maior acesso às informações, percepção de qualificação profissional e conhecimento de objetivos, valores, linguagem e tradição organizacionais.

A esse respeito, deve-se salientar a importância da percepção de suporte social nos processos de adaptação, uma vez que, conforme Polleto e Koller (2006), não é simplesmente o meio socioambiental que confere a qualidade de fator de proteção, mas as significações internas do indivíduo dadas a ele. De acordo com tais significações, o indivíduo pode ou não solicitar apoio aos colegas e, consequentemente, irá ou não contar com o apoio das relações sociais para a sua resiliência e, também, nesse caso, para facilitar a sua socialização.

Retomando, por sua vez, o papel desempenhado pelo fator de resiliência Percepção de si mesmo, o qual descreve a confiança dos indivíduos nas próprias capacidades, os resultados confirmaram o que alguns estudos já demonstravam (Gruman et al., 2006; Saks, 1995) acerca da relevância do senso de eficácia para a compreensão dos processos organizacionais, reforçando a importância de se trabalhar a autoeficácia dos iniciantes.

No que se refere às influências negativas do fator de Coesão Familiar, tem-se a confirmação dos efeitos indesejáveis do apoio de familiares nos resultados da socialização organizacional, mesmo entre servidores com tempo superior a seis meses de serviço. Esta última constatação permite levantar um questionamento acerca dos limites de validade da noção de que o apoio de familiares repercute apenas 
no estágio inicial da socialização (Louis, 1980; Settoon \& Adkins, 1997). Novas pesquisas podem contribuir para observar se há replicação desses achados.

Ainda, quanto ao papel significativo desempenhado pelas variáveis de nacionalidade e ocupação, cumpre tecer algumas considerações. Diferentes resultados de socialização foram explicados pela nacionalidade, indicando a possibilidade de algumas influências culturais nesse processo. $\mathrm{O}$ menor Acesso às Informações e conhecimento da Linguagem e Tradição entre os servidores da universidade brasileira, por exemplo, pode estar relacionado à maior distância de poder, típica da sociedade brasileira (Hofstede \& Hofstede, 2005) e que envolve desigualdades na distribuição de poder, ocasionando a detenção de conhecimento e informação.

No que tange à Integração às Pessoas, maior entre os servidores da universidade norueguesa, a confiança interpessoal pode ser um aspecto cultural que contribua para explicar tal resultado. Inglehart e Baker (2000) já haviam observado que a maioria das sociedades historicamente protestantes apresenta níveis de confiança interpessoal mais elevados do que as sociedades historicamente católicas, mesmo após o controle da variável de desenvolvimento econômico. Assim, as condições socioeconômicas da Noruega, aliadas à sua tradição religiosa, podem estar reforçando a tendência de confiar mais nos outros e atuando como um dos aspectos facilitadores da maior integração social entre os servidores noruegueses.

A maior Integração à Organização entre os novos servidores da universidade brasileira, por sua vez, pode estar sendo influenciada pela orientação tradicional presente no Brasil (Inglehart \& Baker, 2000). Os itens de conhecimento dos processos e da cultura organizacional, presentes no fator de Integração à Organização indicam, de certa forma, um acolhimento dos padrões organizacionais. Desse modo, é possível que a orientação tradicional brasileira, que enfatiza o respeito à autoridade e a conformidade social (Inglehart \& Wezel, 2005), esteja contribuindo para os comportamentos de aceitação e conformidade ao statu quo, por parte daqueles servidores.

Referindo-se à ocupação, os achados foram de que os docentes tenderam a relatar maior Competência e Proatividade e a conhecerem melhor os Objetivos e Valores Organizacionais, comparativamente aos funcionários técnico-administrativos. Todavia, quando as análises foram conduzidas para o conjunto de novos servidores de cada universidade, separadamente, melhores resultados de socialização por parte de docentes foram verificados apenas entre os novos servidores brasileiros. Isto pode ser um indicador da adoção de práticas sociais mais equitativas pelos noruegueses e mais desiguais pelos brasileiros, nas relações de trabalho. Mais uma vez, o aspecto cultural relativo à maior distância de poder no Brasil pode estar influenciando tais resultados e se refletindo numa menor homogeneidade de socialização entre os servidores docentes e técnicoadministrativos na universidade brasileira.

Por fim, comparando-se a capacidade preditiva da resiliência em relação à socialização organizacional entre os novos servidores das duas instituições, observou-se que esta foi maior entre aqueles lotados na universidade brasileira, corroborando a suposição de que tal relação se daria de forma diferenciada em contextos culturais distintos. Tais achados sugerem que, não obstante as diferenças de cultura organizacional e nacional, a resiliência demonstrou exercer papel relevante nos resultados de socialização, residindo, nesse aspecto, a principal contribuição do estudo.

Não se tem a pretensão de julgar, contudo, que os achados aqui obtidos forneçam respostas definitivas. Novos estudos envolvendo, por exemplo, a aplicação de entrevistas semiestruturadas, ou o desenvolvimento de grupos focais, desenvolvidos junto a organizações de outros segmentos e contextos culturais, podem proporcionar diferentes ângulos de observação do fenômeno. Espera-se, portanto, que os resultados aqui apresentados inspirem outros pesquisadores na busca de entendimento mais claro acerca da relação entre a resiliência dos iniciantes e o processo de socialização organizacional.

Artigo recebido em 18.11.2010. Aprovado em 30.05.2011. 


\title{
Nota
}

\begin{abstract}
${ }^{1} \mathrm{O}$ tamanho da amostra utilizada na realização das primeiras análises de regressão foi calculado seguindo os procedimentos recomendados por Tabachnick e Fidel (2007), de que para a utilização de um conjunto de 8 variáveis antecedentes e de controle seria necessária uma amostra mínima de 114 respondentes. Assim, tendo sido composta por 135 servidores, a amostra atendeu aos critérios de proporção entre o número de participantes e o de variáveis antecedentes e de controle empregadas.
\end{abstract}

\section{Referências}

Ashford, S. J., \& Black, J. S. (1996). Proactivity during organizational entry: the role of desire for control. Journal of Applied Psychology, 81(2), 199-214. doi: 10.1037/0021-9010.81.2.199

Ashforth, B. E., Sluss, D. M., \& Saks, A. M. (2007). Socialization tactics, proactive behavior and newcomer learning. Journal of Vocational Behavior, 70(3), 447-462. doi: 10.1016/j.jvb.2007.02.001

Bauer, T., Bodner, T., Erdogan, B., Truxillo, D. M., \& Tucker, J. S. (2007). Newcomer adjustment during organizational socialization. Journal of applied psychology, 92(3), 707-721. doi: 10.1037/0021-9010.92.3.707

Bauer, T., Morrison, E., \& Callister, R. (1998). Organizational socialization. In G. Ferris (Org.), Research in personnel and human resource management (pp. 149-214). Greenwich: JAI Press.

Bauer, T., \& Taylor, S. (2001). Toward a globalized conceptualization of organizational socialization. In A. Neil, D. S. Ones, H. K. Sinangil, \& C. Viswesvaran (Orgs.), Handbook of industrial, work and organizational psychology (pp. 409-423). London: Sage.

Beyer, J. M., \& Hannah, D. R. (2002). Building on the past: enacting established personal identities in a new work setting. Organization science, 13(6), 636-652. doi: 10.1287/orsc.13.6.636.495

Borges, L. O., Silva, F. H. V. C., Melo, S. L., Oliveira, A. S., \& Roazzi, A. (2010). Re-construção e validação de um inventário de socialização organizacional. Revista de Administração Mackenzie, 11(4), 4-37. doi: 10.1590/S1678-69712010000400002

Bravo, M. J., Peiró, J. M., Rodriguez, I., \& Whitely, W. T. (2003). Social antecedents of the role stress and career enhancing strategies of newcomers. Work \& Stress, 17(3), 195-217. doi: $10.1080 / 02678370310001625658$

Carr, J. C., Pearson, A. W., Vest, M. J., \& Boyar, S. L. (2006). Prior occupational experience, anticipatory socialization, and employee retention. Journal of management, 32(3), 343-359. doi: $10.1177 / 0149206305280749$

Chao, G., O’Leary-Kelly, A., Wolf, S., Klein, H., \& Gardner, P. (1994). Organizational socialization: its content and consequences. Journal of Applied Psychology, 79(5), 730-743. doi: 10.1037/0021-9010.79.5.730

Cooper-Thomas, H. D., \& Anderson, N. (2006). Organizational socialization: a new theoretical model and recommendations for future research and HRM practices in organizations. Journal of managerial psychology, 21(5), 492-516. doi: 10.1108/02683940610673997

Finkelstein, L. M., Kulas, J. T., \& Dages, K. D. (2003). Age differences in proactive newcomer socialization strategies in two populations. Journal of Business and Psychology, 17(4), 473-502. doi: 10.1023/A:1023400201387 
Fraser, M. W., Richman, J. M., \& Galinsky, M. J. (1999). Risk, protection, and resilience: toward a conceptual framework for social work practice. Social Work Research, 23(3), 131-143.

Friborg, O., Barlaug, D., Martinussen, M., Rosenvinge, J., \& Hjemdal, O. (2005). Resilience in relation to personality and intelligence. International Journal of Methods in Psychiatric Research, 14(1), 29-42. doi: 10.1002/mpr.15

Friborg, O., Hjemdal, O., Rosenvinge, J. H., \& Martinussen, M. (2003). A new rating scale for adult resilience. International Journal of Methods in Psychiatric Research, 12(2), 65-76. doi: $10.1002 / \mathrm{mpr} .143$

Gruman, J. A., Saks, A. M., \& Zweig, D. I. (2006). Organizational socialization tactics and newcomer proactive behaviors. Journal of Vocational Behavior, 69(1), 90-104. doi: 10.1016/j.jvb.2006.03.001

Haueter, J. A., Macan, T. H., \& Winter, J. (2003). Measurement of newcomer socialization: construct validation of a multidimensional scale. Journal of Vocational Behavior, 63(1), 20-39. doi: 10.1016/S0001-8791(02)00017-9

Hjemdal, O., Friborg, O., Stiles, T., Rosenvinge, J. H., \& Martinussen, M. (2006). Resilience predicting psychiatric symptoms. Clinical psychology and psychotherapy, 13(3), 194-201. doi: $10.1002 /$ cpp.488

Hjemdal, O., Roazzi, A., Dias, M. G., Roazzi, M., \& Vikan, A. (2009). Exploring the psychometric properties of the RSA in a Brazilian sample. In D. Elizur \& E. Yaniv (Orgs.), Facet new horizons in theory construction and data analysis (pp. 120-138). Jerusalem: FTA.

Hofstede, G., \& Hofstede, G. J. (2005). Cultures and organizations: software of the mind. New York: McGraw-Hill.

Inglehart, R., \& Baker, W. E. (2000). Modernization, cultural change, and the persistence of traditional values. American Sociological Review, 65(1), 19-51. doi: 10.2307/2657288

Inglehart, R., \& Wezel, C. (2005). Modernization, cultural change and democracy: the human development sequence. New York: Cambridge University Press.

Jessor, R., Van Den Bos, J., Vanderryn, J., Costa, F. M., \& Turbin, M. S. (1995). Protective factors in adolescent problem behavior. Developmental psychology, 31(6), 923-933. doi: 10.1037/00121649.31.6.923

Kish, L. (1996). Diseño estadístico para la investigación. Madri: Siglo Veintiuno de España.

Lisbona, A., Morales, J., \& Palací, F. (2009). Engagement as a consequence of organizational socialization. International Journal of Psychology \& Psychological Therapy, 9(1), 89-100.

Louis, M. R. (1980). Surprise and sense making: what newcomers experience in entering unfamiliar organizational settings. Administrative science quarterly, 25(2), 226-251.

Masten, A. (2001). Ordinary magic: resilience processes. American psychologist, 56(3), 227-238. doi: 10.1037/0003-066X.56.3.227

Miller-Loessi, K., \& Parker, J. N. (2006). Cross-cultural social psychology. In J. Delamater (Org.), Handbook of social psychology (pp. 529-553). New York: Springer.

Pinheiro, D. P. N. (2004). A resiliência em discussão. Psicologia em estudo, 9(1), 67-75. doi: 10.1590/S1413-73722004000100009 
Polleto, M., \& Koller, S. H. (2006). Resiliência: uma perspectiva conceitual e histórica. In D. D. Dell'Aglio, S. H. Koller, \& M. A. M. Yunes (Orgs.), Resiliência e psicologia positiva: interfaces do risco a proteção (pp. 19-44). São Paulo: Casa do Psicólogo.

Reichers, A. E. (1987). An interactionist perspective on newcomer socialization rates. Academy of management review, 12(2), 278-287.

Ros, M., \& Gouveia, V. V. (2006). Validade dos modelos transculturais sobre os valores. In M. Ros \& V. V. Gouveia (Orgs.), Psicologia social dos valores humanos: desenvolvimentos teóricos, metodológicos e aplicados (pp. 207-235). São Paulo: Senac.

Rutter, M. (1999). Resilience concepts and findings. Journal of family therapy, 21(2), 119-144. doi: $10.1111 / 1467-6427.00108$

Saks, A. M. (1995). Longitudinal field investigation of the moderating and mediating effects of selfefficacy on training and adjustment. Journal of applied psychology, 80(2), 211-225. doi: 10.1037/0021-9010.80.2.211

Saks, A. M., \& Ashforth, B. E. (1997). Organizational socialization: making sense of past and present as a prologue for the future. Journal of vocational behavior, 51(2), 234-279. doi: 10.1006/jvbe.1997.1614

Sanzovo, C. E., \& Coelho, M. E. C. (2007). Estressores e estratégias de coping em uma amostra de psicólogos clínicos. Estudos de psicologia, 24(2), 227-238. doi: 10.1590/S0103166X2007000200009

Scarparo, H. (2000). Psicologia e pesquisa. Porto Alegre: Sulina.

Settoon, R. P., \& Adkins, C. L. (1997). Newcomer socialization: the role of supervisors, coworkers, friends and family members. Journal of Business and Psychology, 11(4), 507-516. doi: 10.1007/BF02195895

Tabachnick, B. G., \& Fidell, L. S. (2007). Using multivariate statistics. Boston: Pearson.

Taormina, R. J. (1997). Organizational socialization: a multidomain, continuous process models. International Journal of Selection and Assessment, 5(1), 29-47. doi: 10.1111/1468-2389.00043

Triandis, H. C. (1994). Culture and social behavior. New York: McGraw-Hill.

Vergara, S. C. (2008). A resiliência de profissionais angolanos. Revista de Administração Pública, 42(4), 701-718. doi: 10.1590/S0034-76122008000400004

Wanberg, C. R., \& Kammeyer-Mueller, J. D. (2000). Predictors and outcomes of proactivity in the socialization process. Journal of Applied Psychology, 85(3), 373-385. doi: 10.1037/00219010.85.3.373 